\title{
Proteome-wide cellular thermal shift assay reveals novel crosstalk between brassinosteroid and auxin signaling
}

Qing $\mathrm{Lu}^{1,2}$, Yonghong Zhang ${ }^{1,2, \dagger}$ Joakim Hellner ${ }^{3}$, Xiangyu Xu ${ }^{1,2}$, Jarne Pauwels ${ }^{4,5}$, Caterina Giannini ${ }^{6}$, Qian $\mathrm{Ma}^{1,2}$, Wim Dejonghe ${ }^{1,2}$, Huibin Han ${ }^{6, \ddagger}$, Brigitte Van de Cotte ${ }^{1,2}$,Francis Impens ${ }^{4,5,7}$, Kris Gevaert ${ }^{4,5}$, Jiří Friml $^{6}$, Ive De Smet ${ }^{1,2}$, Daniel Martinez Molina ${ }^{3}$, Eugenia Russinova ${ }^{1,2, *}$

${ }^{1}$ Department of Plant Biotechnology and Bioinformatics, Ghent University; 9052 Ghent, Belgium; ${ }^{2}$ Center for Plant Systems Biology, VIB; 9052 Ghent, Belgium; ${ }^{3}$ Pelago Bioscience AB, 17148 Solna, Sweden; ${ }^{4}$ Department of Biomolecular Medicine, Ghent University; 9052 Ghent, Belgium; ${ }^{5}$ Center for Medical Biotechnology, VIB; 9052 Ghent, Belgium; ${ }^{6}$ Institute of Science and Technology Austria, 3400 Klosterneuburg, Austria; ${ }^{7}$ VIB Proteomics Core; 9052 Ghent, Belgium; ${ }^{\dagger}$ Current address: School of Basic Medicine, Hubei University of Medicine; Shiyan 442000, China; ${ }^{\star}$ Current address: Research Center for Plant functional genes and Plant Tissue Culture Technology, College of Bioscience and Bioenginering, Jiangxi Agricultural University; Nanchang 330045, Jiangxi, China.

Corresponding author: Eugenia Russinova

Email: eurus@psb.vib-ugent.be

Author Contributions: W. D., D. M. M., and E. R. initiated the work. Q. L., Y. Z., W. D., and E. R. designed the experiments. Q. L. and Y. Z. did CETSA and cloning. Q. L. did the in vitro kinase assays. Q. L., W. D., and D. M. M. performed CESTA-MS and J. H. and D. M. M. analyzed the data. Y. Z., X. X., B. V. C., and I. D. S. did the phosphoproteomics and analyzed data. J. P., F. I., and K. G. did the shotgun proteomics, analyzed data, and provided guidance. H. H., C. G., and J. F. contributed PIN immunolocalizations. Q. L. and E. R. wrote the manuscript. All authors commented on the results and the manuscript.

Competing Interest Statement: D.M.M. is the inventor of patents related to the CETSA method and is a cofounder, board member and employee of Pelago Bioscience AB. No other authors have competing interests.

Keywords: Cellular thermal shift assay, chemical genetics, brassinosteroids, auxin 


\begin{abstract}
Despite the growing interest in using chemical genetics in plant research, small-molecule target identification remains a major challenge. The cellular thermal shift assay coupled with high-resolution massspectrometry (CETSA MS) that monitors changes in the thermal stability of proteins caused by their interactions with small molecules, other proteins, or post-translational modifications allows the identification of drug targets, or the study of protein-metabolite and protein-protein interactions mainly in mammalian cells. To showcase the applicability of this method in plants, we applied CETSA MS to intact Arabidopsis thaliana cells and identified the thermal proteome of the plant-specific glycogen synthase kinase 3 (GSK3) inhibitor, bikinin. A comparison between the thermal- and the phospho-proteomes of bikinin revealed the auxin efflux carrier PIN-FORMED1 (PIN1) as a novel substrate of the Arabidopsis GSK3s that negatively regulate the brassinosteroid signaling. We established that PIN1 phosphorylation by the GSK3s is essential for maintaining its intracellular polarity that is required for auxin-mediated regulation of vascular patterning in the leaf thus, revealing a novel crosstalk between brassinosteroid and auxin signaling.
\end{abstract}




\section{Significance Statement}

Chemical genetics, which investigates the biological processes using small molecules, is gaining interest in plant research. However, a major challenge is to uncover the mode of action of the small molecule. Here, we applied the cellular thermal shift assay coupled with mass spectrometry (CETSA MS) to intact Arabidopsis cells and showed that bikinin, the plant-specific glycogen synthase kinase 3 (GSK3) inhibitor, changed the thermal stability of some of its direct targets and putative GSK3 interacting proteins. In combination with phosphoproteomics, we also revealed that GSK3s phosphorylate the auxin carrier PINFORMED1 (PIN1) and regulated its polarity that is required for the vascular patterning in the leaf. 


\section{Introduction}

Although the application of chemical genetics to plant research is gaining interest (1-3), unravelling the mode of action of the small molecules remains a major challenge. The cellular thermal shift assay (CETSA) is a label-free method that can assess target engagement directly in live cells (4), but its application to plant cells remains limited $(5,6)$. The technique is based on the biophysical principle that a ligand can induce changes in the thermal stability of the target protein, allowing the generation of so-called protein melting curves $(7,8)$. Similarly to the classical thermal shift assay with purified proteins, small-molecule binding typically leads to protein stabilization and an increase in melting temperature $\left(\mathrm{T}_{\mathrm{m}}\right)$. Coupling CETSA with multiplexed quantitative mass spectrometry (MS) enables the monitoring of an entire proteome for changes in protein thermostability in the presence of a small molecule $(9,10)$. Consequently, proteins interacting with this molecule can be identified without previous knowledge of the pathways or molecular mechanisms involved (11). As the thermal stability of a protein may also be affected by post-translational modifications or by binding to other proteins, cofactors, or metabolites, CETSA MS carried out on intact cells, in which active signaling takes place, allows the identification of effector proteins downstream of the direct target (9).

The small molecule bikinin (12) is an inhibitor specifically targeting the Arabidopsis thaliana Shaggy/ glycogen synthase kinase 3 (GSK3)-like kinases (AtSKs), including the key negative brassinosteroid (BR) signaling regulator BR-INSENSITIVE2 (BIN2)/AtSK21 that phosphorylates and inactivates two main transcription factors, BRASSINAZOLE RESISTANT1 (BZR1) and BRI1-EMSSUPPRESSOR1 (BES1)/BZR2 (13, 14). The Arabidopsis genome encodes 10 AtSKs, which belong to four groups (15) evidence exists for at least six, AtSK11, AtSK12, AtSK13, AtSK22, AtSK23, and AtSK32, that they negatively regulate the BR signaling just as BIN2/AtSK21 (16-18). Likewise the mammalian GSK3s (19), AtSKs also phosphorylate numerous substrates and control many developmental and physiological processes in plants such as root, stomatal and flower development, xylem differentiation, responses to light, and different abiotic and biotic stresses (15). BIN2/AtSK21 also mediates the crosstalk between BR and other plant hormones, including auxin (15).

In spite of the reported interdependency and cooperation (20-25), the molecular mechanisms of the signaling crosstalk between BRs and auxin are still not well understood. Although auxin does not affect the phosphorylation state of the BZR1 and BES1/BZR2 transcription factors (14), BIN2/AtSK21 interacts and phosphorylates the AUXIN RESPONSE FACTOR2 (ARF2) (21). This phosphorylation results in a loss in the DNA binding and repressor activity of ARF2 and facilitates auxin responses (21). BIN2/AtSK21 also 
phosphorylates and activates ARF7 and ARF19 to promote lateral root development through an increase in auxin response (22). Moreover, BRs have been shown to control posttranscriptionally the endocytic sorting of PIN-FORMED 2 (PIN2) (23) and stimulate the nuclear abundance and signaling of auxin via repressing the accumulation of PIN-LIKES (PILS) proteins at the endoplasmic reticulum (24).

Here, by adapting the CETSA MS to Arabidopsis intact cells and combining it with the phosphoproteomics, we discovered that the auxin efflux carrier PIN-FORMED1 (PIN1) is a novel substrate of the AtSKs. We found that phosphorylation mediated by the AtSKs is required for PIN1 polarity and for leaf venation. In summary, we demonstrate that CETSA MS is a powerful method for identification of small-molecule targets as well as for discovery of new protein-protein interactions in plant cells.

\section{Results}

\section{CETSA monitoring of small molecule-protein interactions in intact Arabidopsis cells}

Previously, we had used the Western blot-based CETSA for small-molecule target validation in cell lysates of Arabidopsis seedlings (6). To extend the use of CETSA to intact plant cells, we adapted the available protocol (10) to Arabidopsis cell suspension cultures (Fig. S1). First, we tested whether the plant cell wall complicated the protein isolation by evaluating the efficiency of the freeze-thaw lysis method applied for mammalian cells (10). After washing and resuspension in protein extraction buffer, $100-\mu 1$ aliquots of cells were freeze-thawed multiple times and the protein concentration of the supernatant was measured with the Bradford protein assay. The protein concentration in the lysate plateaued at the seventh freeze-thaw cycle (Fig. S2A). Moreover, considering that plants grow over a wider temperature range, we assessed whether in intact Arabidopsis cells proteins follow a melting profile similar to that in lysates when heated $(5,26)$. To this end, we heated 100- $\mu 1$ aliquots of Arabidopsis cells in protein extraction buffer were heated to different temperatures (from $25^{\circ} \mathrm{C}$ to $80^{\circ} \mathrm{C}$ ) for $2 \mathrm{~min}$ and analyzed the lysates by sodium dodecyl-sulfate polyacrylamide gel electrophoresis (SDS-PAGE) after seven freeze-thaw cycles and centrifugation. As expected, the Arabidopsis proteins unfolded and precipitated at high temperature (Fig. S2 $B$ and $C$ ).

Subsequently, as a proof of concept, we aimed to apply CETSA to cells treated with bikinin, an inhibitor of several AtSKs in Arabidopsis (12). Application of bikinin at concentrations of 30-50 $\mu \mathrm{M}$ to Arabidopsis seedlings induces BR responses that can be measured by changes in the phosphorylation status of the transcription factor BES1/BZR2 (14). To check whether bikinin is effective in Arabidopsis cell suspension cultures we treated cells with $50 \mu \mathrm{M}$ bikinin for $30 \mathrm{~min}$. As expected, bikinin induced the dephosphorylation of BES1 in the cell cultures similarly to the most active BR, brassinolide (BL) (Fig. S2D). Taken together, bikinin can induce BR responses in Arabidopsis cell suspension cultures. 
Next, we investigated the effect of bikinin on the thermal stability of its direct targets, the ten AtSKs (12), by means of the adjusted CETSA protocol (Fig. S1) and by using Western blots for detection. In brief, after 30 min of treatment with bikinin or dimethylsulfoxide (DMSO), the Arabidopsis cells were washed, resuspended in protein extraction buffer containing either bikinin or DMSO, and aliquoted into PCR tubes. Then, the aliquots were heated to 12 distinct temperatures $(30,35,40,43,46,49,52,55,58,61,65$, and $70^{\circ} \mathrm{C}$ ) for $2 \mathrm{~min}$. Afterward, the heated cells were lysed through seven freeze-thaw cycles followed by Western blot-based protein detection. As specific antibodies for all AtSKs are not available, Arabidopsis cell suspension cultures overexpressing the hemagglutinin (HA)-tagged AtSKs were utilized to generate protein-melting curves and to assess the bikinin-induced $\mathrm{T}_{\mathrm{m}}$ shifts. First, we examined whether bikinin induced $\mathrm{T}_{\mathrm{m}}$ shifts for AtSK12 and AtSK13 at a $50-\mu \mathrm{M}$ concentration, but, surprisingly, found that it stabilized AtSK13 with a $\mathrm{T}_{\mathrm{m}}$ shift of $5.94^{\circ} \mathrm{C}$ (Fig. S3A), but not AtSK12 (Fig. S3B). By contrast, the thermal stability of the ATP synthase $\beta$ (ATP $\beta$ ), used as a control, was not affected by bikinin. We further determined the half-maximum effective concentration $\left(\mathrm{EC}_{50}\right)$ of bikinin for AtSK12 by means of isothermal doseresponse fingerprinting (ITDRF $\mathrm{CETSA}_{\text {) }}$ ) at $45^{\circ} \mathrm{C}$ to ensure a sufficient shift in the denaturation temperature. At low concentrations, bikinin did not affect the thermal stability of AtSK12 and the relative band intensity reached a plateau at approximately $250 \mu \mathrm{M}$ (Fig. S3C). Therefore, to ensure saturation and achieve sufficiently sized $\mathrm{T}_{\mathrm{m}}$ shifts for all AtSKs, we used $250 \mu \mathrm{M}$ bikinin (Fig. 1). Staining of the cells with the cell viability tracer, fluorescein diacetate (FDA), excluded the potential cytotoxic effect of bikinin when used at high concentrations (Fig. S2E). Of the 10 putative bikinin targets (12), AtSK11, AtSK12, AtSK13, BIN2/AtSK21, AtSK22, and AtSK41 showed $\mathrm{T}_{\mathrm{m}}$ shifts, whereas the thermal stability of AtSK23, AtSK31, AtSK32, and AtSK42 was not affected by the small molecule (Fig. 1) as well as the thermal denaturation of the ATP $\beta$ control (Fig. S4). Collectively, these results showed that bikinin stabilized most of its targets, indicating that the CETSA protocol was applicable to intact Arabidopsis cells.

\section{CETSA MS of bikinin in intact Arabidopsis cells}

Several recent studies in mammalian cells reported the use of proteome-wide CETSA MS for obtaining a comprehensive view on small molecule-protein interactions through determination of individual temperature shifts $(8-10,27)$. Therefore, we extended the CETSA protocol as described above (Fig. S1) to the Arabidopsis proteome (Fig. S2D) by using $50 \mu \mathrm{M}$ bikinin, the BR response-inducing concentration in cell cultures. Briefly, following the heating at $25,30,35,40,45,50,55,60,70$, and $80^{\circ} \mathrm{C}$ and freeze-thaw cycles, samples were analyzed with a nanoscale liquid chromatography coupled to tandem mass spectrometry (nano LC-MS/MS), whereafter they were digested with trypsin and labeled with 10-plex tandem mass tag (TMT10). 
In total, 6,000 proteins were identified of which the melting profiles were defined for 4,225 proteins in samples both treated with bikinin and DMSO (Dataset S1A). Approximately $96 \%$ of the identified proteins had a melting temperature within the range of $35^{\circ} \mathrm{C}-60^{\circ} \mathrm{C}$ (Fig. $2 A$ and Dataset $\mathrm{S} 1 A$ ), of which only 61 proteins displayed a significant change in thermal stability $(27$ were stabilized and 34 were destabilized) in the presence of $50 \mu \mathrm{M}$ bikinin (absolute value of $\mathrm{T}_{\mathrm{m}}$ shift $\geq 2{ }^{\circ} \mathrm{C}$, analysis of variance (ANOVA)-based F-test $P<0.01$ ) (Fig. $2 B$ and Dataset S1). However, because none of the AtSKs was identified by the CETSA MS, we examined the protein expression of AtSKs in Arabidopsis cell suspension cultures. Although quantitative reverse transcription PCR (qRT-PCR) revealed expression of all AtSKs in the cell cultures (Fig. S5A), only AtSK11, AtSK21, AtSK31 and AtSK41, albeit at a low intensity were detected with shotgun proteomics (Fig. S5B and Dataset S2). Given that all AtSKs were efficiently extracted using the CETSA protocol when overexpressed (Fig. 1), probably the low number of peptides identifying these proteins in cell cultures obstructed the creation of their melting curves (Dataset S2).

As changes in thermal stability had been observed also for downstream effectors of the direct smallmolecule target, possibly as a result of altered posttranslational modifications or interactions with other proteins $(4,9)$, we examined whether any of the 61 proteins $(P<0.01)$ (Dataset $S 2 B)$ functioned together with the AtSKs and found that only the MITOGEN-ACTIVATED PROTEIN KINASE3 (MPK3); which acts downstream of the known BIN2/AtSK21 interactor YODA (YDA) (28), exhibited a $\mathrm{T}_{\mathrm{m}}$ shift $\left(-2.96^{\circ} \mathrm{C}\right.$, $P<0.01)$. Furthermore, we checked whether any of the 61 proteins with significant $\mathrm{T}_{\mathrm{m}}$ shifts were putative AtSK interactors (Fig. S6) according to the STRING database (29). Based on this analysis, the HOPW1-1INTERACTING2 (WIN2, AT4G31750) $\left(\mathrm{T}_{\mathrm{m}} \mathrm{shift}=7.80{ }^{\circ} \mathrm{C}, P<0.01\right)$ (Fig. $2 \mathrm{C}$ ) was selected as a possible AtSK-interacting protein. Interaction between HA-tagged WIN2 and each of the 10 GFP-tagged AtSKs was observed by co-immunoprecipitation experiments carried out using tobacco (Nicotiana tabacum) cells transiently overexpressing the proteins (Fig. 2D). In summary, in cell suspension cultures bikinin affected the thermal stability of the Arabidopsis proteome and induced $\mathrm{T}_{\mathrm{m}}$ shifts in several proteins that might be putative AtSK-interacting proteins or AtSK downstream effectors.

\section{The phosphoproteome of bikinin}

As, besides MPK3, the proteins with altered thermal stability identified by the CETSA MS (Dataset S1) were neither direct bikinin targets nor known downstream AtSK effectors, we carried out a phosphoproteomics analysis on bikinin-treated Arabidopsis cell suspension cultures. We hypothesized that the putative $A t$ SK-interacting proteins or AtSK downstream effectors might modify their phosphorylation state upon inhibition of the kinase activity of the AtSKs. Arabidopsis cell suspension cultures were treated with $50 \mu \mathrm{M}$ bikinin or DMSO for $30 \mathrm{~min}$, under the same CETSA MS. The phosphoproteomics analysis 
revealed that, in total, 9,351 phosphopeptides that were mapped to 1,751 proteins (Dataset S3A). Bikinin treatment significantly down regulated $(P<0.05)$ the phosphorylation intensities of 972 phospho-sites that belong to 665 proteins (Fig. 3A, Fig. S7 and Dataset S3 B-E), of which, six proteins were known AtSKinteracting proteins including, BZR1 (30), ARF2 (21), YDA (28), GLUCOSE-6-PHOSPHATE DEHYDROGENASE6 (G6PD6) (31), TETRATRICOPETIDE-REPEAT THIOREDOXIN-LIKE3 (TTL3) (32), and OCTOPUS (33). In addition, the phosphorylation intensities of 101 phospho-sites belonging to 84 proteins were significantly up regulated $(P<0.05)$ (Fig. $3 A$, Fig. S7 and Dataset S3 $B-E$ ). Of note, both downregulated phosphosites and upregulated phosphosites were identified in 35 proteins (Fig $3 A$ and Dataset S3 $B-E$ ). The gene ontology (GO) enrichment analysis (34) showed that most of the enriched terms for the identified phosphorylation-regulated proteins were related to mRNA splicing, metabolic process, and transport (Fig. S8A, Dataset S3G). However, of the bikinin-regulated phosphoproteins, only six (phosphorylation-downregulated) proteins showed significant $\mathrm{T}_{\mathrm{m}}$ shifts in the CETSA MS (Dataset $\mathrm{S} 3 E$ ), including the auxin efflux carrier PIN1 $\left(\mathrm{T}_{\mathrm{m}} \mathrm{shift}=3.83^{\circ} \mathrm{C}\right)($ Fig. $3 A$ and $B$ ). Taken together, only a few proteins identified in the bikinin phosphoproteome displayed bikinin-induced changes in their thermal stability.

\section{AtSKs phosphorylate PIN1 and regulate its polarity}

We hypothesized that PIN1 is a direct substrate of the AtSKs, because the PIN1 protein had been identified in the CETSA MS (Fig. $3 B$ and $D$, and Dataset S1) and the phosphorylation intensities of five residues in the hydrophilic loop (HL) (Ser ${ }^{271}, \mathrm{Ser}^{282}, \mathrm{Thr}^{286}, \mathrm{Ser}^{290}$, and $\mathrm{Ser}^{337}$ ) (Fig. S9) were reduced in the presence of $50 \mu \mathrm{M}$ bikinin (Fig. $3 C$ and Dataset S3C). To verify this observation, we carried out a protein kinase assay by incubating the polyhistidine (HIS)-tagged hydrotropic loop (HL) of PIN1 with HIS-small ubiquitin-like modifier (HIS-SUMO)-tagged AtSK proteins in an in vitro phosphorylation reaction. The results showed that eight AtSK proteins phosphorylated the His-PIN1-HL (Fig. 4A). Remarkably, four of the five PIN1 residues, of which the phosphorylation was altered by the bikinin, had previously been identified as targets of the serine/threonine protein kinases PINOID/AGCVIII kinases (PID/WAGs) (35), D6 Protein Kinases (D6PKs) (36), and MPKs (MPK3, MPK4, and MPK6) (37, 38). Moreover, these residues were conserved (Fig. S9) and essential for the polar localization of the PIN proteins with long HLs (39).

Given the prominent role of phosphorylation in regulating polarity, we next assessed whether treatments with bikinin and BL affected the polar localization of PIN1 and PIN2 in the root meristem by means of immunolocalization. Wild type Arabidopsis plants were treated in liquid medium with $50 \mu \mathrm{M}$ bikinin, $10 \mathrm{nM} \mathrm{BL}$, and $0.1 \%$ (v/v) DMSO for $12 \mathrm{~h}$. As anticipated, PIN1 displayed a more apolar 
localization in the presence of bikinin and BL (Fig. $4 B$ and $C$ ) than with DMSO, whereas the PIN2 polarity remained unaffected (Fig. S10). Given that PIN1-mediated polar auxin transport regulates the foliar vascular patterning (40), we examined whether AtSKs were involved in the regulation of leaf venation. Five-day-old wild type Arabidopsis plants, germinated and grown in liquid medium containing $50 \mu \mathrm{M}$ bikinin and $10 \mathrm{nM}$ $\mathrm{BL}$, had an abnormal vascular patterning in the cotyledons, mainly with missing loops, when compared to the mock control (Fig. S11). To provide genetic evidence for AtSK function in leaf venation, we analyzed the vascular pattern of the AtSK quadruple (atsk13RNAi bin2 bill bil2, designated atsk ${ }^{\text {quad }}$ ) and sextuple (atsk11RNAi atsk12RNAi atsk13RNAi bin2 bill bil2, designated atsk ${ }^{\text {sext }}$ ) mutants (41). Consistent with BR and bikinin treatments, the atsk ${ }^{\text {quad }}$ mutant exhibited an abnormal vascular patterning, with missing loops and disconnected upper loops (Fig. $4 D$ and $E$ ). Because of the seedling lethality of the atsk ${ }^{\text {sext }}$ mutant (41), the plant phenotypes were analyzed in the $\mathrm{T} 1$ generation. The atsk $k^{\text {sext }}$ mutant also displayed an abnormal vascular patterning, but mainly with missing loops (Fig. $4 D$ and $E$ ).

To test whether the identified five phosphorylation sites were relevant for the AtSK-mediated regulation of PIN1, we generated phospho-inactive His-PIN1-HL ${ }^{5 \mathrm{~A}}$ by substituting the Thr and Ser residues with Ala. The phosphorylation of His-PIN1-HL ${ }^{5 \mathrm{~A}}$ by BIN2/AtSK in vitro was reduced but not abolished (Fig. S12A), suggesting that more sites were phosphorylated by BIN2/AtSK21. Next, we introduced the phospho-inactive PIN1 ${ }^{5 \mathrm{~A}}$-GFP (PIN1pro::PIN1 ${ }^{5 \mathrm{~A}}$-GFP) into wild type Arabidopsis plants. Noteworthy, eight of 21 GFP-expressing transgenic plants had naked inflorescence stems in the first-generation (Fig. S12B), reminiscent of the pin1 null mutant (42). Then, we examined the localization of PIN1 ${ }^{5 \mathrm{~A}}-\mathrm{GFP}$ in the root tip cells of wild type-resembling transgenic plants. When expressed at the same levels as the native PIN1-GFP ( $p$ PIN1::PIN1-GFP) (43) (Fig. S12C), PIN1 ${ }^{5 \mathrm{~A}}$-GFP exhibited a more apolar localization in two independent transgenic lines (Fig. $4 F$ and $G$ ) that, similarly way to the atsk $k^{\text {quad }}$ and atsk $^{\text {sext }}$ mutants, had an abnormal vascular patterning in the cotyledons (Fig. $4 H$ and $I$ ). In summary, these observations show that AtSK-mediated phosphorylation of PIN1 is important for their polar localization and for leaf venation.

\section{Discussion}

Given the challenges in small-molecule target identification in plants (44), novel experimental strategies, especially label-free methods, are required to facilitate chemical genetics studies. CETSA is one such method that has been proven useful for the detection of direct drug targets and downstream effects of druginduced perturbations in several cellular systems and tissues $(9,27,45,46)$. In plants, CETSA has been successfully applied for small-molecule target validation in cell lysates of Arabidopsis (6) and, recently, the 
thermal profiles of more than 2,000 proteins in Arabidopsis lysates have been reported (5, 26). Here, we explored the potential of CETSA to identify the protein targets of the AtSK kinase inhibitor bikinin (12) in intact Arabidopsis cells. In Western blot-CETSA, bikinin stabilized six of the ten AtSKs. As expected, bikinin had no effect on either AtSK31 or AtSK42, of which the kinase activity was not inhibited in vitro (12), but surprisingly, on AtSK23 and AtSK32 as well, of which the in vitro inhibition of the kinase activity had previously been reported (12). One reason might be that the binding affinity of bikinin to AtSK23 and AtSK32 is lower than that to other AtSKs, thus requiring higher bikinin concentrations to induce the noticeable $\mathrm{T}_{\mathrm{m}}$ shifts. Moreover, in a previous study, approximately $30 \%$ of the target proteins of the promiscuous kinase inhibitor staurosporine did not show $\mathrm{T}_{\mathrm{m}}$ shifts (9), suggesting that some protein kinases are not responsive in CETSA.

By combining CETSA with MS, we identified the thermal profiles of 4,225 proteins in the presence of bikinin in Arabidopsis cell suspension cultures, of which 61 (1.44\%) showed significant $\mathrm{T}_{\mathrm{m}}$ shifts (absolute value $\geq 2{ }^{\circ} \mathrm{C}$, ANOVA-based F-test $P<0.01$ ). Although the CETSA MS assay did not detect any AtSKs, probably due to their low protein abundance in the cell cultures, MPK3, the substrate of the know BIN2/AtSK21 interactor YDA (28), was identified. In addition, the thus far unknown interaction between WIN2 (AT4G31750) and AtSKs was validated in vivo. WIN2 is implicated in the modulation of the defense responses of induced by the Pseudomonas syringae effector protein HopW1-1 (47). AtSK11 has been shown to regulate the pattern-triggered immunity and the susceptibility to P. syringae, probably through G6PD6 phosphorylation (48). Our data suggest that AtSK11, as well as its homologs, might regulate the immune responses to $P$. syringae via direct interaction with WIN2.

To demonstrate the capability of CESTA MS to identify proteins that might bind the AtSKs directly or function in downstream pathways, we carried out a phosphoproteomics analysis in the presence of bikinin. Phosphorylation sites in 665 proteins were downregulated upon bikinin treatment, comprising six known substrates of the AtSKs, hence, confirming the quality of our data. Noteworthy, the phosphorylation of 84 proteins was upregulated upon bikinin treatment, indicating that these proteins might be indirect AtSK targets. The GO enrichment analysis for the bikinin-regulated phosphoproteins revealed that AtSKs might be involved in the regulation of RNA splicing and protein intracellular transport, like their mammalian homologs $(49,50)$. Moreover, of all proteins with differential phosphorylation, 76 proteins were also identified by proximity labelling with BIN2/AtSK21 as a bait (51) (Dataset S3F). Thus, we speculate that some of these proteins might be novel substrates of the AtSKs. By comparing the CETSA MS and phosphoproteome datasets, we found that the thermal stability of most of these proteins was not affected, even though their phosphorylation intensities were altered by the bikinin treatment. A possible reason might be that either the phosphorylation cannot affect the protein melting behavior or that the in the bikinin- 
induced changes in the phosphorylation intensity are not sufficient to induce an important $\mathrm{T}_{\mathrm{m}}$ shift for most of these proteins. However, for a few proteins, bikinin had an impact on both the phosphorylation and melting behaviors, indicating that the phosphorylation status might affect the thermal stability of some proteins. Although some studies in human cells reported that phosphorylation affected the protein thermal stability (52), others demonstrated that for most of the proteins, the melting behavior of phosphorylated and non-phosphorylated forms were concordant $(53,54)$.

Particularly, bikinin reduced the phosphorylation and induced the thermal stabilization of the auxin efflux carrier PIN1. The identified phosphorylation sites in the HL of PIN1 by AtSKs are conserved among the PIN proteins (Fig. S9), some of which are essential for their polar localization and the intercellular auxin transport (39). However, although some kinases shared phosphorylation sites in the PIN proteins, for instance PID/WAGs and D6PKs, their corresponding mutants or transgenic overexpression lines affected the PIN polarities differently $(35,36)$. Moreover, the auxin-regulated receptor CANALIZATIONRELATED AUXIN-REGULATED MALECTIN-TYPE RECEPTOR-LIKE KINASE (CAMEL) (55) and CALCIUM-DEPENDENT PROTEIN KINASE 29 (CPK29) (56) phosphorylate PIN1 and controls its polarity via phosphorylation sites that are not shared with other kinases, including PID/WAGs, D6PKs, MPKs and the AtSKs (Fig. S9) $(55,56)$. Therefore, multiple parallel mechanisms for the maintenance of the PIN polarities and activities exists. Our data imply that BRs control the PIN1 phosphorylation and polarity via AtSKs. Two of the five phosphorylation sites identified in our phosphoproteomics study are also targeted by PID/WAGs and D6PKs, whereas another two phosphorylation sites are targeted by MPK3, MPK4, and MPK6, implying a functional redundancy of AtSKs with other kinases in the PIN phosphorylation. In addition, bikinin and BR treatments, which inhibit the activity of AtSKs directly and indirectly, respectively, impaired the PIN1 polarity, indicating that AtSKs control the polarity of PINs in combination with other kinases. Considering this overlap in the PIN1 phosphorylation sites, understanding of the precise environmental or developmental conditions and upstream pathways that coordinate these kinases will be needed to cooperatively control the PIN polarities. Furthermore, because the PIN phosphorylation induced by D6PKs and PID/WAGs control their activity (57), it will be necessary to examine whether bikinin and exogenous BRs disrupt the auxin transport activity of PIN1 and other PINs. Previous studies have shown that BRs affect PIN proteins through different mechanisms $(23,58)$, including transcriptional activation of of PIN4 and PIN7 (58). Additionally, BRs and bikinin stabilized PIN2, but not PIN1, to respectively interfere with the auxin distribution in gravistimulated roots (23) and BRs partially altered PIN2 polarity via the actin cytoskeleton (59). However, others demonstrated that the actin cytoskeleton was not required for the polar localization of PIN2 (60). Interestingly, BRs did not affect the PIN2 polarity in our study, 
although four of the five identified phosphorylation sites are conserved in the HL of PIN2. Together, these data indicate that the mechanisms controlling the polarity and the stability of PINs by BRs differ.

PIN1 has been shown to be expressed in early leaf veins and margins and PIN1-mediated polar auxin transport to be important for the leaf venation pattern in Arabidopsis (40). Moreover, MPK6 could regulate the leaf venation pattern probably by regulating the PIN1 phosphorylation and polarity, although the main MPK6-targeted phosphorylation site $\mathrm{Ser}^{337}$, also targeted by AtSKs, was not involved (37). In addition, the auxin-regulated receptor CAMEL controls the cotyledon venation through modulation of the PIN1 phosphorylation and respectively polarization (55). Our data showed that chemical activity inhibition or genetic knockout of the AtSKs resulted in defective cotyledon venation pattern, suggesting that BRs control leaf venation via AtSK-mediated phosphorylation of PIN1. It remains to be established whether the PIN1 polarity in the veins is affected under these conditions.

In summary, we adapted the CETSA MS method for intact Arabidopsis cells and demonstrated its ability to identify direct targets and downstream components of the small-molecule target proteins. Such information is useful to understand the mode of action of the small molecules and the function of their target proteins. Moreover, we identified PIN1 as a novel substrate of AtSKs, uncovering an unknown BR mechanism and auxin crosstalk.

\section{Materials and Methods}

Details on the material and experimental procedures used in this study (i.e., plant material, CETSA and CETS MS assays, phosphoproteomics, generation and transformation of constructs, confocal microscopy, in vitro kinase assay, whole-mount in situ immunolocalization of PIN1, immunoprecipitation and statistical analysis) can be found in SI Materials and Methods.

\section{Acknowledgments}

We thank Yanhai Yin for providing the anti-BES1 antibody, Johan Winne and Brenda Callebaut for synthesizing bikinin, Yuki Kondo and Hiroo Fukuda for published materials, and Martine De Cock for help in preparing the manuscript.

\section{References}

1. G. R. Hicks, N. V. Raikhel, Small Molecules Present Large Opportunities in Plant Biology. Annual Review of Plant Biology 63, 261-282 (2012). 
2. G. R. Hicks, N. V. Raikhel, Plant chemical biology: are we meeting the promise? Front. Plant Sci. 5 (2014).

3. V. Halder, E. Russinova, Understanding the language of drugged plants. Nature Chemical Biology 15, 1025-1028 (2019).

4. D. Martinez Molina, P. Nordlund, The Cellular Thermal Shift Assay: A Novel Biophysical Assay for In Situ Drug Target Engagement and Mechanistic Biomarker Studies. Annual Review of Pharmacology and Toxicology 56, 141-161 (2016).

5. J. D. Volkening, K. E. Stecker, M. R. Sussman, Proteome-wide analysis of protein thermal stability in the model higher plant Arabidopsis thaliana. Molecular \& Cellular Proteomics, mcp.RA118.001124 (2018).

6. W. Dejonghe, et al., Disruption of endocytosis through chemical inhibition of clathrin heavy chain function. Nat. Chem. Biol. (2019) https:/doi.org/10.1038/s41589-019-0262-1.

7. D. Martinez Molina, et al., Monitoring Drug Target Engagement in Cells and Tissues Using the Cellular Thermal Shift Assay. Science 341, 84-87 (2013).

8. R. Jafari, et al., The cellular thermal shift assay for evaluating drug target interactions in cells. Nature Protocols 9, 2100-2122 (2014).

9. M. M. Savitski, et al., Tracking cancer drugs in living cells by thermal profiling of the proteome. Science 346, 1255784 (2014).

10. H. Franken, et al., Thermal proteome profiling for unbiased identification of direct and indirect drug targets using multiplexed quantitative mass spectrometry. Nature Protocols 10, 1567-1593 (2015).

11. K. V. M. Huber, et al., Proteome-wide drug and metabolite interaction mapping by thermal-stability profiling. Nature Methods 12, 1055-1057 (2015).

12. B. De Rybel, et al., Chemical Inhibition of a Subset of Arabidopsis thaliana GSK3-like Kinases Activates Brassinosteroid Signaling. Chemistry \& Biology 16, 594-604 (2009).

13. Z.-Y. Wang, et al., Nuclear-Localized BZR1 Mediates Brassinosteroid-Induced Growth and Feedback Suppression of Brassinosteroid Biosynthesis. Developmental Cell 2, 505-513 (2002).

14. Y. Yin, et al., BES1 Accumulates in the Nucleus in Response to Brassinosteroids to Regulate Gene Expression and Promote Stem Elongation. Cell 109, 181-191 (2002).

15. C. Li, B. Zhang, H. Yu, GSK3s: nodes of multilayer regulation of plant development and stress responses. Trends Plant Sci. S1360-1385(21)00204-1 (2021).

16. T.-W. Kim, et al., Brassinosteroid signal transduction from cell-surface receptor kinases to nuclear transcription factors. Nature Cell Biology 11, 1254-1260 (2009).

17. Z. Yan, J. Zhao, P. Peng, R. K. Chihara, J. Li, BIN2 Functions Redundantly with Other Arabidopsis GSK3-Like Kinases to Regulate Brassinosteroid Signaling. Plant Physiology 150, 710-721 (2009).

18. W. Rozhon, J. Mayerhofer, E. Petutschnig, S. Fujioka, C. Jonak, ASK $\theta$, a group-III Arabidopsis GSK3, functions in the brassinosteroid signalling pathway. The Plant Journal 62, 215-223 (2010).

19. P. Patel, J. R. Woodgett, "Chapter Eight - Glycogen Synthase Kinase 3: A Kinase for All Pathways?" in Current Topics in Developmental Biology, Protein Kinases in Development and Disease., A. Jenny, Ed. (Academic Press, 2017), pp. 277-302.

20. A. Nakamura, et al., Brassinolide Induces IAA5, IAA19, and DR5, a Synthetic Auxin Response Element in Arabidopsis, Implying a Cross Talk Point of Brassinosteroid and Auxin Signaling. Plant Physiology 133, 1843-1853 (2003). 
21. G. Vert, C. L. Walcher, J. Chory, J. L. Nemhauser, Integration of auxin and brassinosteroid pathways by Auxin Response Factor 2. PNAS 105, 9829-9834 (2008).

22. H. Cho, et al., A secreted peptide acts on BIN2-mediated phosphorylation of ARFs to potentiate auxin response during lateral root development. Nature Cell Biology 16, 66-76 (2014).

23. K. Retzer, et al., Brassinosteroid signaling delimits root gravitropism via sorting of the Arabidopsis PIN2 auxin transporter. Nat Commun 10, 1-15 (2019).

24. L. Sun, et al., PIN-LIKES Coordinate Brassinosteroid Signaling with Nuclear Auxin Input in Arabidopsis thaliana. Current Biology 30, 1579-1588.e6 (2020).

25. M. Ackerman-Lavert, et al., Auxin requirements for a meristematic state in roots depend on a dual brassinosteroid function. Current Biology (2021) https:/doi.org/10.1016/j.cub.2021.07.075 (September 5, 2021).

26. A. Jarzab, et al., Meltome atlas - thermal proteome stability across the tree of life. Nature Methods 17, 495-503 (2020).

27. A. Mateus, et al., Thermal proteome profiling in bacteria: probing protein state in vivo. Mol. Syst. Biol. 14, e8242 (2018).

28. T.-W. Kim, M. Michniewicz, D. C. Bergmann, Z.-Y. Wang, Brassinosteroid regulates stomatal development by GSK3-mediated inhibition of a MAPK pathway. Nature 482, 419-422 (2012).

29. D. Szklarczyk, et al., STRING v10: protein-protein interaction networks, integrated over the tree of life. Nucleic Acids Res 43, D447-D452 (2015).

30. J.-X. He, J. M. Gendron, Y. Yang, J. Li, Z.-Y. Wang, The GSK3-like kinase BIN2 phosphorylates and destabilizes BZR1, a positive regulator of the brassinosteroid signaling pathway in Arabidopsis. PNAS 99, 10185-10190 (2002).

31. S. D. Santo, et al., Stress-Induced GSK3 Regulates the Redox Stress Response by Phosphorylating Glucose-6-Phosphate Dehydrogenase in Arabidopsis. The Plant Cell 24, 3380-3392 (2012).

32. V. Amorim-Silva, et al., TTL Proteins Scaffold Brassinosteroid Signaling Components at the Plasma Membrane to Optimize Signal Transduction in Arabidopsis. The Plant Cell 31, 1807-1828 (2019).

33. P. Anne, et al., OCTOPUS Negatively Regulates BIN2 to Control Phloem Differentiation in Arabidopsis thaliana. Current Biology 25, 2584-2590 (2015).

34. S. X. Ge, D. Jung, R. Yao, ShinyGO: a graphical gene-set enrichment tool for animals and plants. Bioinformatics 36, 2628-2629 (2020).

35. J. Friml, et al., A PINOID-Dependent Binary Switch in Apical-Basal PIN Polar Targeting Directs Auxin Efflux. Science 306, 862-865 (2004).

36. I. C. R. Barbosa, M. Zourelidou, B. C. Willige, B. Weller, C. Schwechheimer, D6 PROTEIN KINASE Activates Auxin Transport-Dependent Growth and PIN-FORMED Phosphorylation at the Plasma Membrane. Developmental Cell 29, 674-685 (2014).

37. W. Jia, et al., Mitogen-Activated Protein Kinase Cascade MKK7-MPK6 Plays Important Roles in Plant Development and Regulates Shoot Branching by Phosphorylating PIN1 in Arabidopsis. PLOS Biology 14, e1002550 (2016).

38. M. Dory, et al., Coevolving MAPK and PID phosphosites indicate an ancient environmental control of PIN auxin transporters in land plants. FEBS Letters 592, 89-102 (2018).

39. I. C. R. Barbosa, U. Z. Hammes, C. Schwechheimer, Activation and Polarity Control of PINFORMED Auxin Transporters by Phosphorylation. Trends in Plant Science 23, 523-538 (2018). 
40. E. Scarpella, D. Marcos, J. Friml, T. Berleth, Control of leaf vascular patterning by polar auxin transport. Genes Dev. 20, 1015-1027 (2006).

41. Y. Kondo, et al., Plant GSK3 proteins regulate xylem cell differentiation downstream of TDIF-TDR signalling. Nature Communications 5 (2014).

42. T. Vernoux, J. Kronenberger, O. Grandjean, P. Laufs, J. Traas, PIN-FORMED 1 regulates cell fate at the periphery of the shoot apical meristem. Development 127, 5157-5165 (2000).

43. E. Benková, et al., Local, Efflux-Dependent Auxin Gradients as a Common Module for Plant Organ Formation. Cell 115, 591-602 (2003).

44. W. Dejonghe, E. Russinova, Plant Chemical Genetics: From Phenotype-Based Screens to Synthetic Biology. Plant Physiology 174, 5-20 (2017).

45. J. M. Dziekan, et al., Identifying purine nucleoside phosphorylase as the target of quinine using cellular thermal shift assay. Science Translational Medicine 11, eaau3174 (2019).

46. J. Perrin, et al., Identifying drug targets in tissues and whole blood with thermal-shift profiling. Nature Biotechnology 38, 303-308 (2020).

47. M. W. Lee, J. Jelenska, J. T. Greenberg, Arabidopsis proteins important for modulating defense responses to Pseudomonas syringae that secrete HopW1-1. The Plant Journal 54, 452-465 (2008).

48. H. Stampfl, M. Fritz, S. D. Santo, C. Jonak, The GSK3/Shaggy-Like Kinase ASK $\alpha$ Contributes to Pattern-Triggered Immunity. Plant Physiology 171, 1366-1377 (2016).

49. A. Adachi, et al., Golgi-associated GSK3 regulates the sorting process of post-Golgi membrane trafficking. Journal of Cell Science 123, 3215-3225 (2010).

50. M. Y. Shinde, et al., Phosphoproteomics reveals that glycogen synthase kinase-3 phosphorylates multiple splicing factors and is associated with alternative splicing. Journal of Biological Chemistry 292, 18240-18255 (2017).

51. T.-W. Kim, et al., Application of TurboID-mediated proximity labeling for mapping a GSK3 kinase signaling network in Arabidopsis. bioRxiv, 636324 (2019).

52. J. X. Huang, et al., High throughput discovery of functional protein modifications by Hotspot Thermal Profiling. Nature Methods 16, 894-901 (2019).

53. C. M. Potel, et al., Impact of phosphorylation on thermal stability of proteins. Nat Methods, 1-3 (2021).

54. I. R. Smith, et al., Identification of phosphosites that alter protein thermal stability. Nat Methods $\mathbf{1 8}$, 760-762 (2021).

55. J. Hajný, et al., Receptor kinase module targets PIN-dependent auxin transport during canalization. Science 370, 550-557 (2020).

56. H. Lee, A. Ganguly, S. Baik, H.-T. Cho, CALCIUM-DEPENDENT PROTEIN KINASE 29 modulates PIN-FORMED polarity and Arabidopsis development via its own phosphorylation code. The Plant Cell (2021) https:/doi.org/10.1093/plcell/koab207 (August 18, 2021).

57. M. Zourelidou, et al., Auxin efflux by PIN-FORMED proteins is activated by two different protein kinases, D6 PROTEIN KINASE and PINOID. eLife 3, e02860 (2014).

58. A. Nakamura, H. Goda, Y. Shimada, S. Yoshida, Brassinosteroid Selectively Regulates PIN Gene Expression in Arabidopsis. Bioscience, Biotechnology, and Biochemistry 68, 952-954 (2004).

59. M. Lanza, et al., Role of Actin Cytoskeleton in Brassinosteroid Signaling and in Its Integration with the Auxin Response in Plants. Developmental Cell 22, 1275-1285 (2012). 
60. M. Glanc, M. Fendrych, J. Friml, PIN2 Polarity Establishment in Arabidopsis in the Absence of an Intact Cytoskeleton. Biomolecules 9, 222 (2019).

61. A. Houbaert, et al., POLAR-guided signalling complex assembly and localization drive asymmetric cell division. Nature 563, 574 (2018).

62. Z. Yang, et al., Structure of the Arabidopsis JMJ14-H3K4me3 Complex Provides Insight into the Substrate Specificity of KDM5 Subfamily Histone Demethylases. The Plant Cell (2017) https:/doi.org/10.1105/tpc.17.00666 (January 7, 2020).

63. S. Tan, et al., The lipid code-dependent phosphoswitch PDK1-D6PK activates PIN-mediated auxin efflux in Arabidopsis. Nature Plants 6, 556-569 (2020).

64. H. Liu, J. H. Naismith, An efficient one-step site-directed deletion, insertion, single and multiple-site plasmid mutagenesis protocol. BMC Biotechnology 8, 91 (2008).

65. J. Van Leene, et al., A Tandem Affinity Purification-based Technology Platform to Study the Cell Cycle Interactome in Arabidopsis thaliana. Molecular \& Cellular Proteomics 6, 1226-1238 (2007).

66. M. Sauer, et al., Canalization of auxin flow by Aux/IAA-ARF-dependent feedback regulation of PIN polarity. Genes Dev. 20, 2902-2911 (2006).

67. C. S. Hughes, et al., Ultrasensitive proteome analysis using paramagnetic bead technology. Molecular Systems Biology 10, 757 (2014).

68. L. D. Vu, et al., Up-to-Date Workflow for Plant (Phospho)proteomics Identifies Differential DroughtResponsive Phosphorylation Events in Maize Leaves. J. Proteome Res. 15, 4304-4317 (2016).

69. J. Friml, E. Benková, U. Mayer, K. Palme, G. Muster, Automated whole mount localisation techniques for plant seedlings. The Plant Journal 34, 115-124 (2003). 
bioRxiv preprint doi: https://doi.org/10.1101/2021.12.14.472597; this version posted December 16, 2021. The copyright holder for this preprint (which was not certified by peer review) is the author/funder, who has granted bioRxiv a license to display the preprint in perpetuity. It is made available under aCC-BY-ND 4.0 International license.
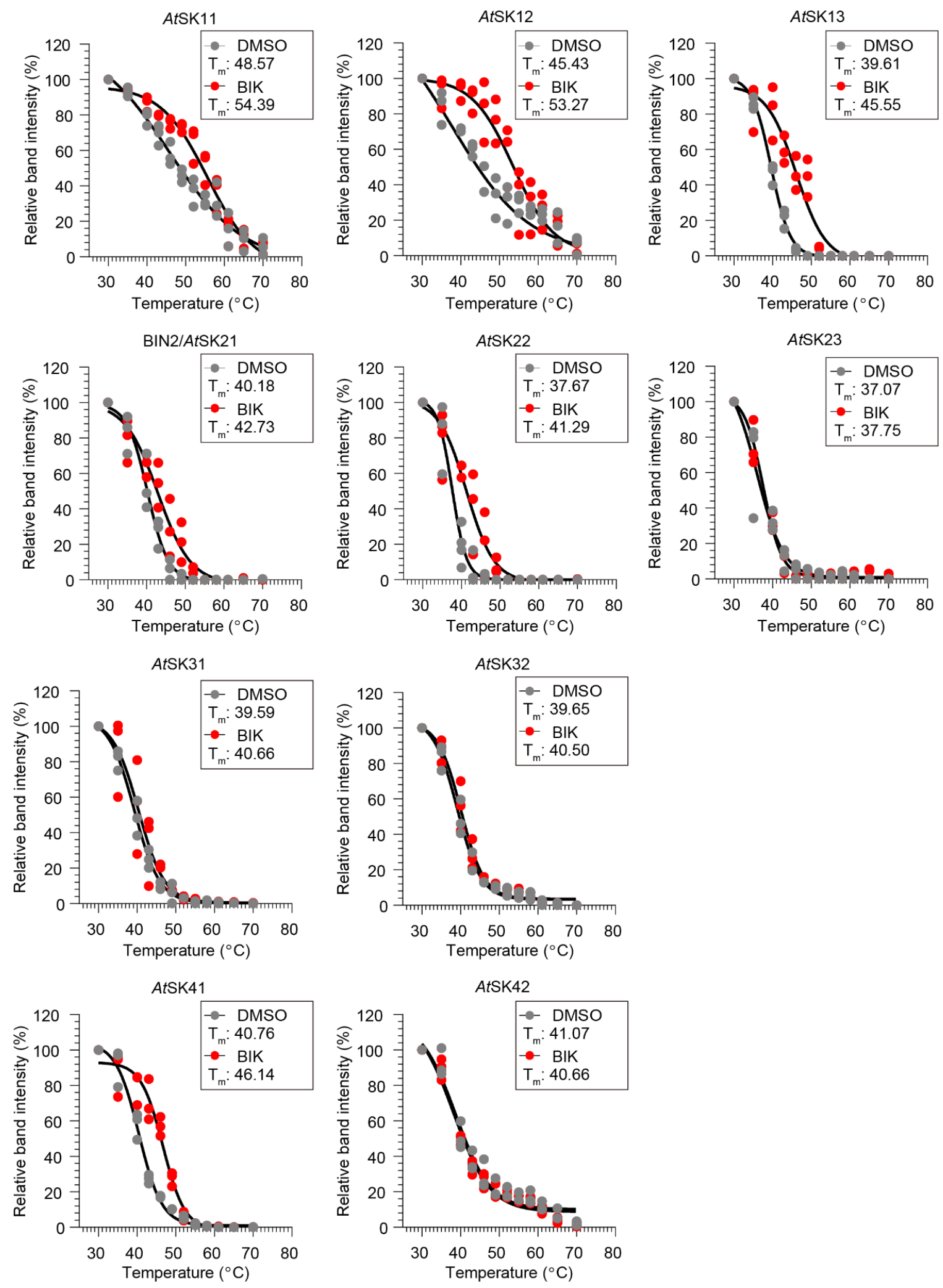
Figure 1. Bikinin stabilized a subset of the AtSKs. Thermal denaturation curves for 10 hemagglutinin (HA)-tagged AtSKs stably overexpressed in Arabidopsis cell suspension cultures in the presence of $250 \mu \mathrm{M}$ bikinin $(\mathrm{BIK})$ or $0.1 \%(\mathrm{v} / \mathrm{v})$ DMSO. The relative band intensities from the Western blot analysis were calculated based on the lowest temperature $\left(30^{\circ} \mathrm{C}\right)$. Melting temperatures $\left(\mathrm{T}_{\mathrm{m}}\right)$ are indicated. Individual data points are plotted for three biological replicates. 

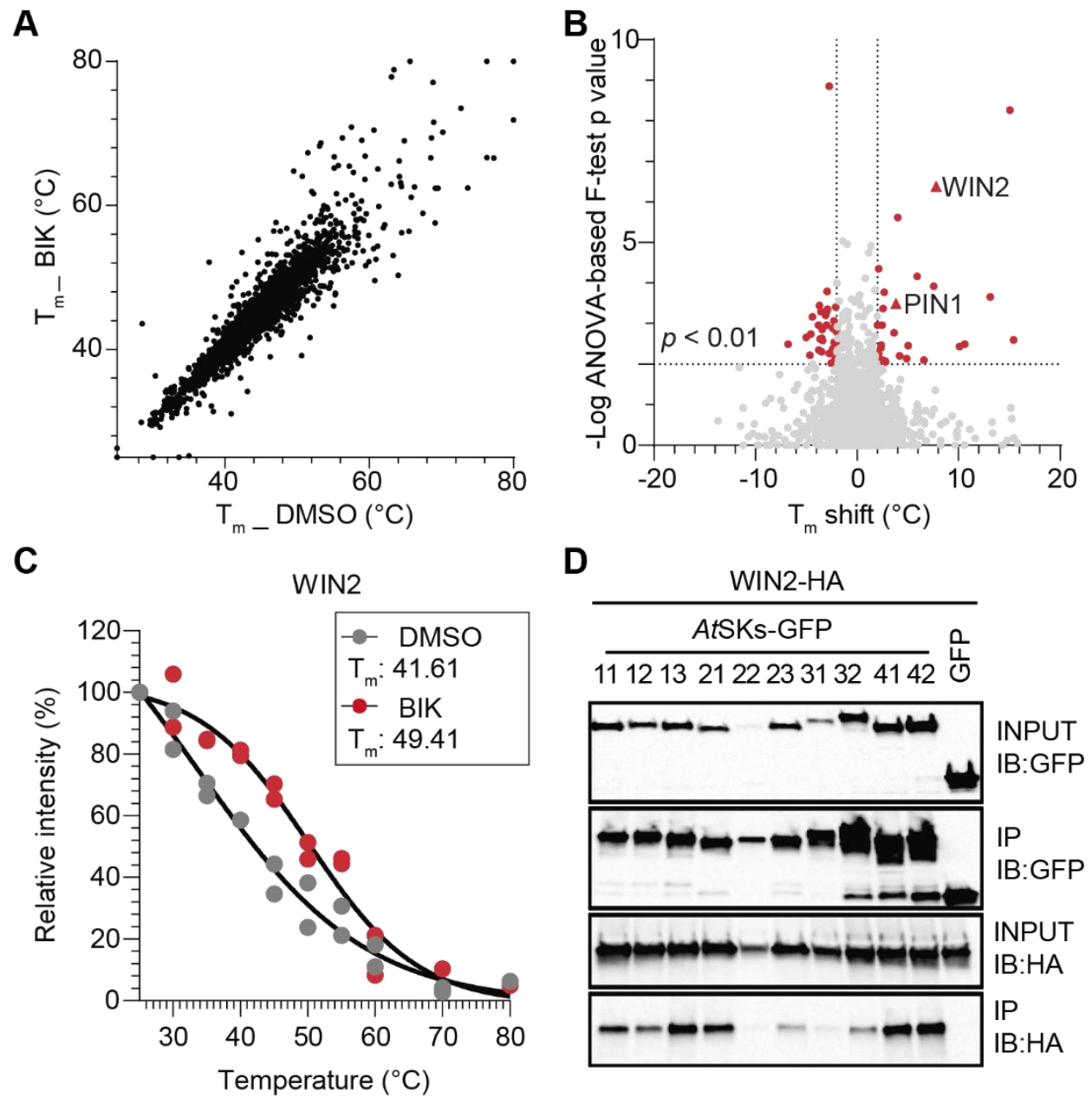

Figure 2. CETSA MS in the presence of bikinin. (A) Melting temperatures of the 4,225 proteins identified in the CETSA MS in the presence of $50 \mu \mathrm{M}$ bikinin (BIK) or $0.1 \%(\mathrm{v} / \mathrm{v})$ DMSO. (B) Distribution of the $\mathrm{T}_{\mathrm{m}}$ shifts of all proteins. Proteins in red highlight significant changes $\left(\mathrm{T}_{\mathrm{m}}\right.$ shift $>2^{\circ} \mathrm{C}$, ANOVA-based F-test $P$ < 0.01). (C) Thermal denaturation curves generated for WIN2 in the presence of $50 \mu \mathrm{M}$ BIK or $0.1 \%(\mathrm{v} / \mathrm{v})$ DMSO subtracted from the CETSA MS in Arabidopsis cell suspension cultures. The $\mathrm{T}_{\mathrm{m}}$ is indicated. Individual data points are plotted for $n=2$ technical replicates. (D) Coimmunoprecipitation of WIN2 with all At SKs when coexpressed in tobacco. The $p 35 S::$ WIN2-HA construct was transiently coexpressed with either p35S::AtSKs-GFP or p35S::GFP (as a negative control). Proteins were extracted (Input) and immunoprecipitated (IP) by means of GFP beads. AtSKs-GFP, GFP, and WIN2-HA were detected with anti-GFP and anti-HA antibody, respectively. IP, immunoprecipitation; IB, immunoblot. 
A

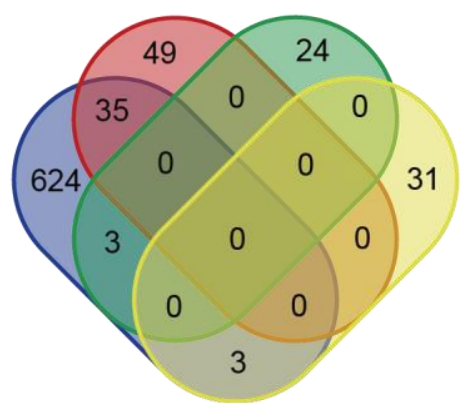

Phosphorylation downregulated proteins Phosphorylation upregulated proteins Stabilized proteins in CETSA-MS Destabilized proteins in CETSA-MS

C

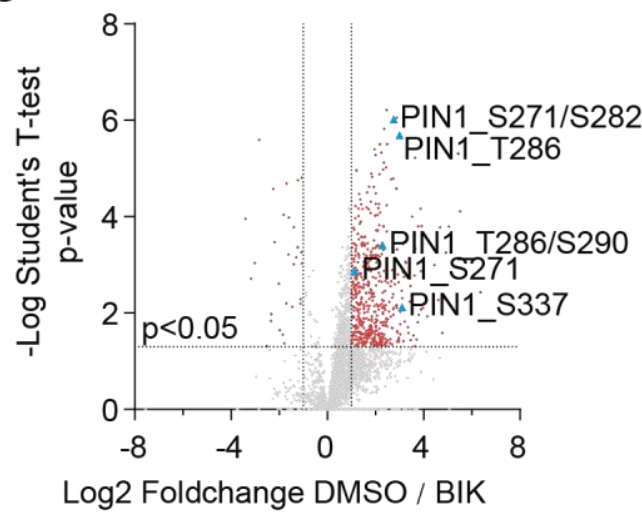

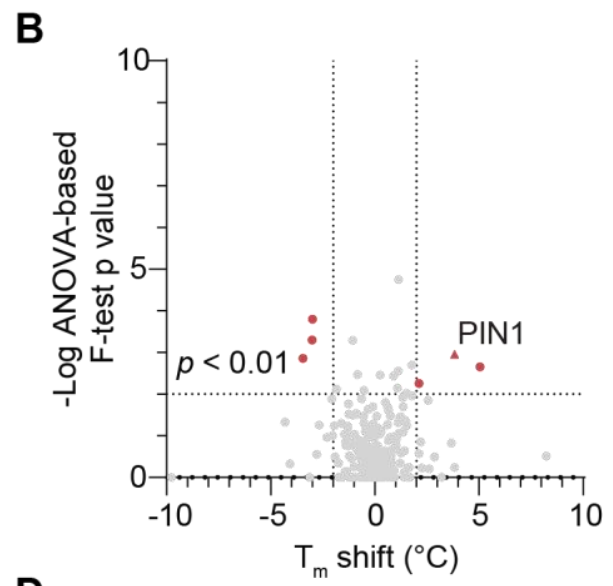

D

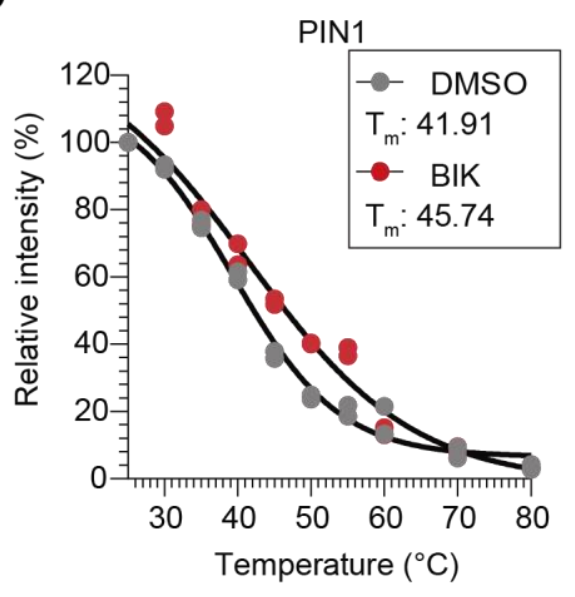

Figure 3. Identification of PIN1. (A) Venn diagram comparing the CETSA MS and the bikinin (BIK) phosphoproteome. Only six proteins (phosphorylation downregulated) showed significant $\mathrm{T}_{\mathrm{m}}$ shifts in CETSA MS. (B) $\mathrm{T}_{\mathrm{m}}$ shifts distribution of the proteins of which the phosphorylation intensities were downregulated in the presence of $50 \mu \mathrm{M}$ BIK. (C) Volcano plot representation of quantitative phosphoproteomics analysis in the presence of $50 \mu \mathrm{M}$ BIK and $0.1 \%$ (v/v) DMSO. Diamonds represent phosphopeptides quantified in five biological replicates, each phosphopeptide log 2 (fold change) is the average logarithmic ratio of phosphopeptide abundance of in cell suspensions treated with BIK vs DMSO plotted against the $\log 10$ ( $P$ value) determined with the Student's $t$ test. Phosphopeptides of PIN1 are indicated. (D) The thermal denaturation curves generated for PIN1 in the presence of $50 \mu \mathrm{M}$ BIK or $0.1 \%$ (v/v) DMSO subtracted from the CETSA MS in Arabidopsis cell suspension cultures. The $\mathrm{T}_{\mathrm{m}}$ is indicated. Individual data points are plotted for $n=2$ technical replicates. 
A

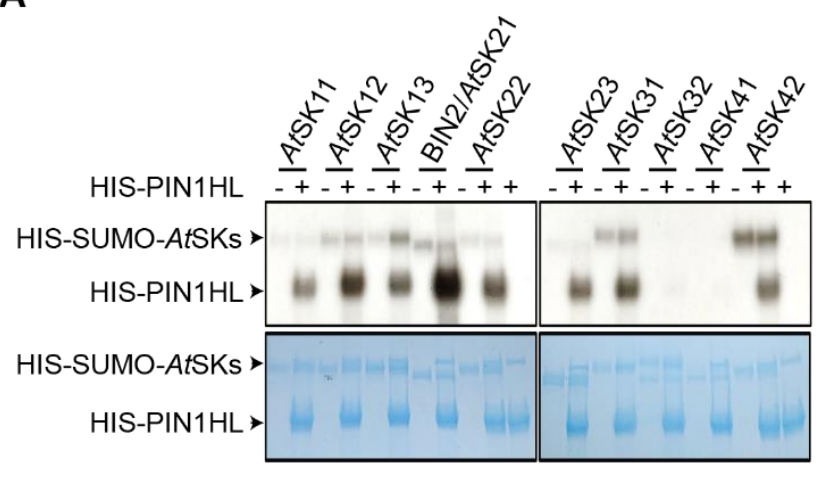

B

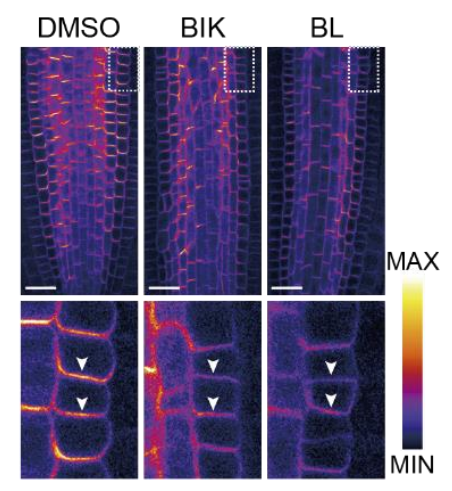

C

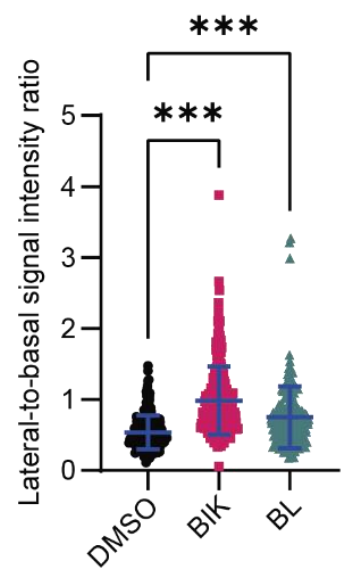

H

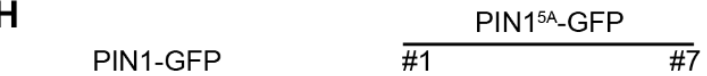

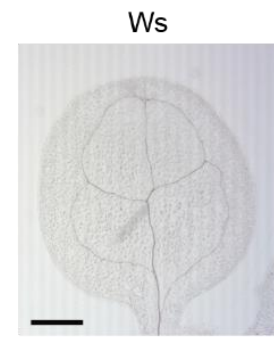

E

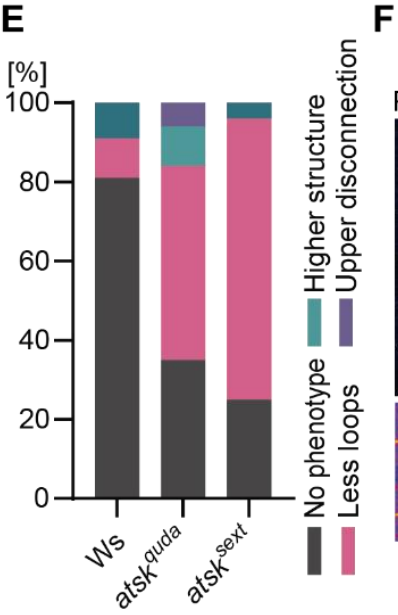

$\mathbf{F}$ atsk qudr
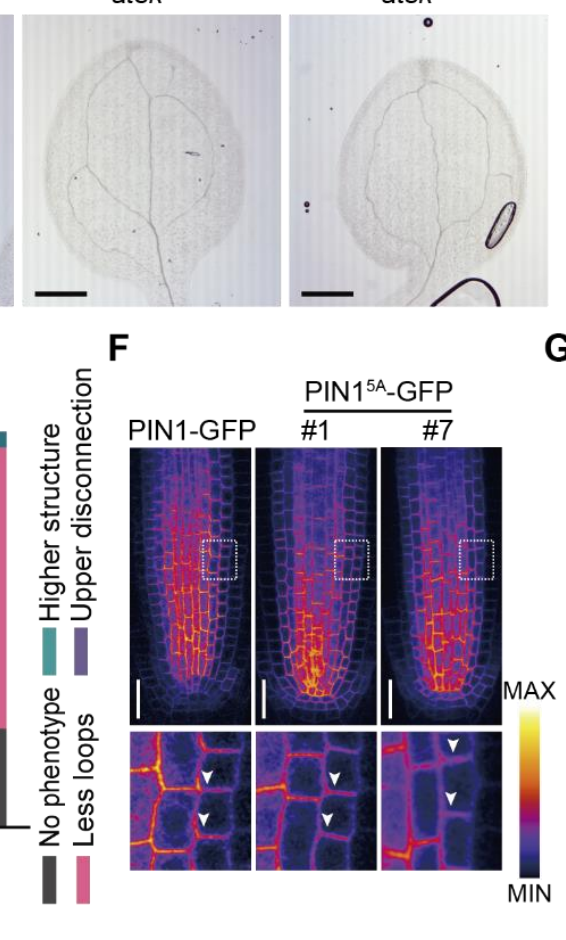

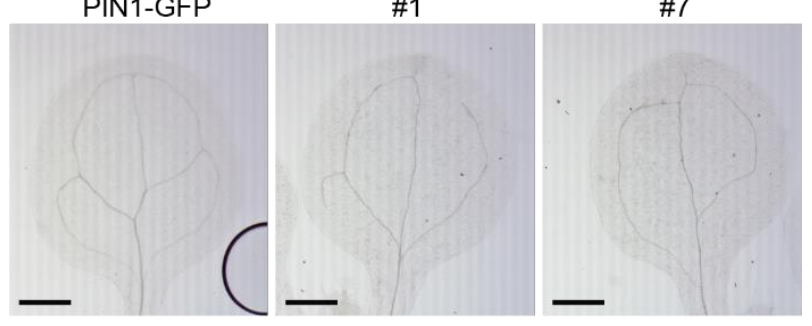

G

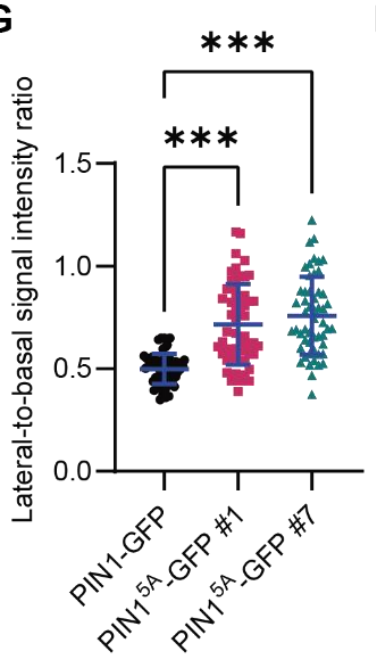

I

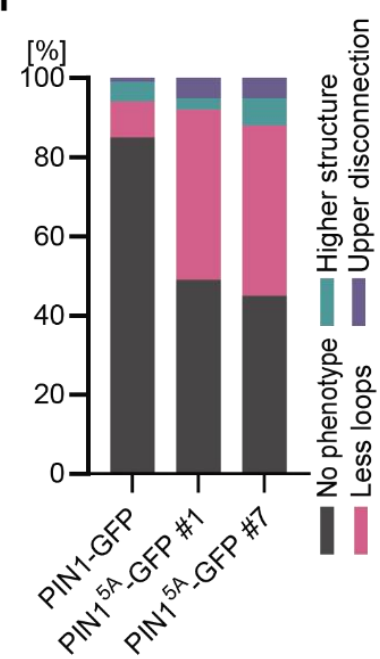

Figure 4. PIN1 phosphorylation and polarity modulation by the AtSKs. (A) Phosphorylation of HIS-

PIN1HL by HIS-SUMO-AtSKs in vitro. The signal of the AtSKs-induced phosphorylation of HIS-PIN1HL were indicated in the autoradiography (Top). The Coomassie Brilliant Blue (CBB) showed the presence of the respective recombinant proteins (bottom). (B) Immunolocalization of PIN1 in root tips after $12 \mathrm{~h}$ of $50 \mu \mathrm{M}$ bikinin (BIK), $10 \mathrm{nM}$ brassinolide (BL), or $0.1 \%$ (v/v) DMSO treatments. The pictures below each main image represent zoomed in regions. Scale bars, $20 \mu \mathrm{m}$. (C) Quantitative evaluation of (B) showing mean of PIN1 lateral-to-basal signal intensity ratio in endodermal cells. $n>180$ cells corresponding to a minimum of 15 roots per treatment from three independent experiments. (D) Representative images of 
venation patterning defects in cotyledons of Wassilewskija (Ws), AtSK quadruple mutant (AtSKs ${ }^{\text {qudr }}$, and sextuple mutant $\left(A t S K^{\text {sext }}\right)$. Scale bars, $1 \mathrm{~mm}$. (E) Quantification of venation defects in $A t \mathrm{SK}^{\text {qudr }}$ and $A t S K^{\text {sext }}$ mutants ( $n>60$ of each genotype from three independent experiments). (F) Subcellular localization of PIN1-GFP and PIN1-GFP ${ }^{5 \mathrm{~A}}$ in root tip cells. The pictures below each main image represent zoomed regions. Arrowheads indicate the lateral membrane signal in the endodermal cells. (G) Quantitative evaluation of (C) showing mean of PIN1-GFP and PIN1-GFP ${ }^{5 \mathrm{~A}}$ lateral-to-basal signal intensity ratio in endodermal cells. $n>60$ cells corresponding to a minimum of 15 roots per genotype from three independent experiments. $(\mathbf{H})$ Representative images of venation patterning defects in cotyledons of PINIpro:: PIN1-GFP and two transgenic lines of PIN1pro::PIN1 ${ }^{5 A}$-GFP. Scale bars, $1 \mathrm{~mm}$. (I) Quantification of venation defects in PIN1pro:: PIN1-GFP and two PIN1pro::PIN1 ${ }^{5 A}$-GFP transgenic lines $(n>60$ of each genotype from three independent experiments). (C and G) Scatter dot plots showing all the individual points with the means and standard errors. One-way ANOVA with Tukey's post hoc test compared to DMSO or PIN2-GFP respectively, $* * * P<0001$. 